\title{
Chest pain with induration of the chest wall
}

\author{
Toshihiko Machi
}

A 39-year-old man was admitted for worsening left pleuritic chest pain of three months duration. He had been diagnosed as having diabetes mellitus six years previously, but glycaemic control had been poor. He was a long-term alcoholic. He did not remember having had any skin lesion or trauma involving the chest wall.

On physical examination, his body temperature was $36.9^{\circ} \mathrm{C}$. His oral hygiene was poor with numerous dental cavities and severe periodontitis. On the left hypochondrial region, a hard subcutaneous induration that was not associated with any superficial skin lesion was felt. Chest auscultation revealed nothing abnormal. White blood cell count was $9.8 \times 10^{9} / 1$. Erythrocyte sedimentation rate was $70 \mathrm{~mm} / \mathrm{h}$, and C-reactive protein was $17.2 \mathrm{mg} / \mathrm{dl}$. Blood biochemistry was completely normal. Postprandial blood glucose level was $380 \mathrm{mg} / \mathrm{dl}$, and glycosylated haemoglobin, HbAlc, was $13.4 \%$. Plain chest X-ray revealed a shallow consolidation in the left lower lung field. Results of plain radiography of the ribs and bone scintigraphy were normal. Computed tomography (CT) disclosed the presence of a left pleural effusion (figure 1 ).

After admission, thoracentesis was performed three times. The effusion had characteristics of an exudate and contained a large percentage of lymphocytes. No atypical cells were observed and no microorganisms grew on aerobic or anaerobic cultures. From day 6, his body temperature increased to more than $38^{\circ} \mathrm{C}$ and the subcutaneous induration continued to increase in size. On day 19 , a purulent liquid, including many grey granules, $1-3 \mathrm{~mm}$ in diameter, was aspirated from the chest wall lesion. Gram staining of smears of the pus and granules is shown in figure 2.
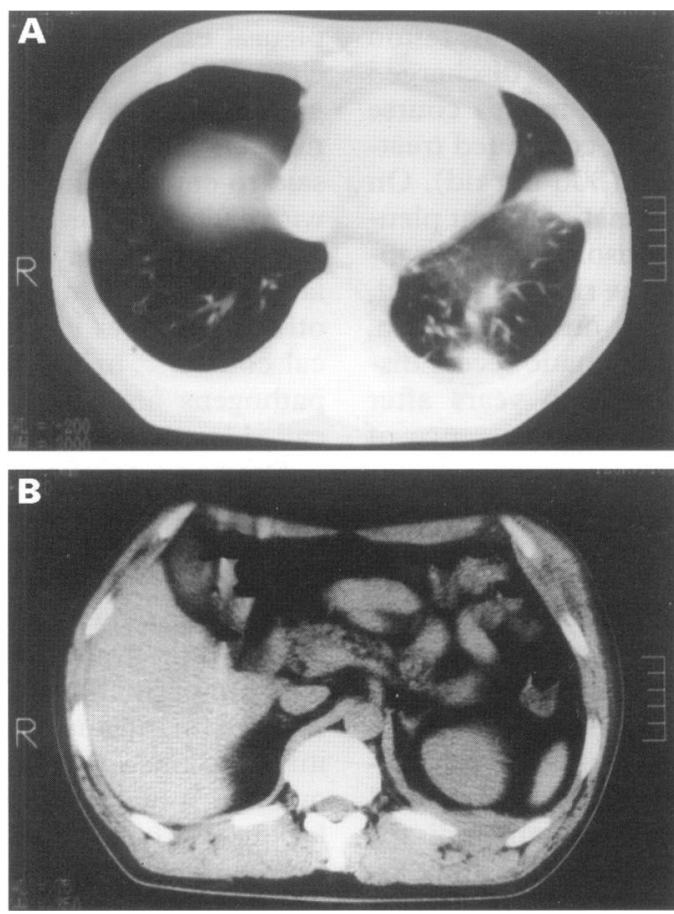

Figure 1 CT scan
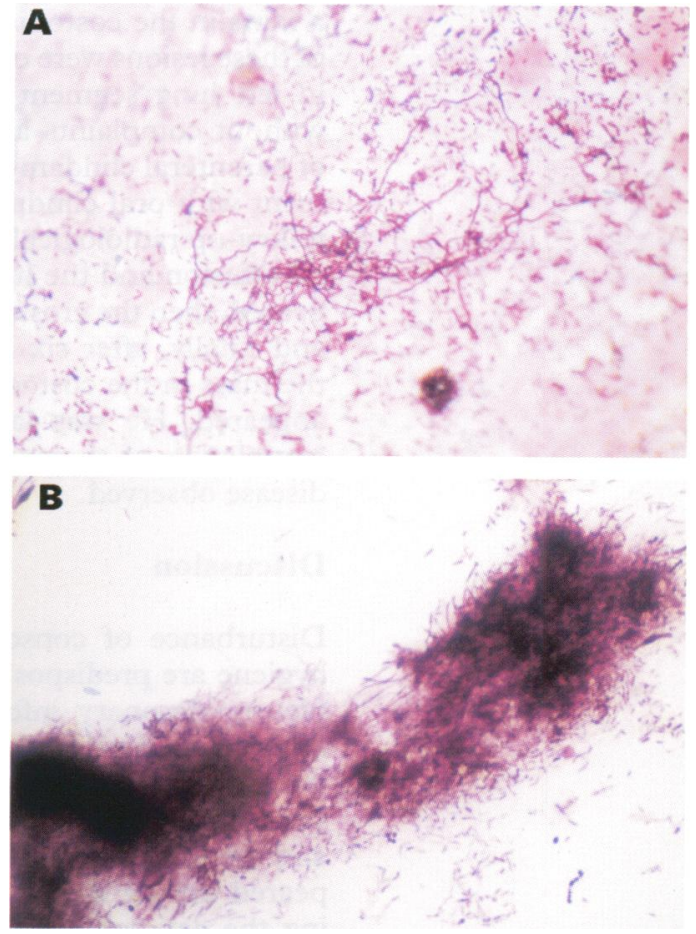

Figure 2 Gram staining of smears of (A) pus, (B) crushed granule (original $\times 200$ )

\section{Department of Internal Medicine, Keiju General Hospital, Tomioka machi, Nanao, Ishikawa, Japan T Machi}

Accepted 20 August 1997

\section{Questions}

1 What does the CT scan (figure 1) show?

2 What does the Gram staining of smears (figure 2) show?

3 What is the diagnosis? 


\section{Answers}

QUESTION 1

The CT scan (figure 1) shows thickened lesions in the left hypochondrial wall and the left posterior pleura, showing continuity with the lung parenchymal lesions in segments 8 and 10 , respectively.

QUESTION 2

Gram-positive cocci, negative rods and filamentous branching positive rods were present in the pus (figure $2(\mathrm{~A})$ ). The granules consisted mainly of filamentous rods (figure $2(B)$ ).

QUESTION 3

The diagnosis was thoracic actinomycosis.

\section{Clinical course}

An incision was made to drain the abscess and the patient was started on parenteral clindamycin (600 mg tid). The fever abated within a few days. Five species of anaerobes were cultured from the pus, all of them sensitive to clindamycin. The anaerobes were identified as Actinomyces israelii, Peptostreptococcus micros, Fusobacterium $\mathrm{sp}$, Bacteroides oris and Bacteroides sp. Beginning a few days after the incision, when the patient was afebrile, he complained of dull pain in the left infrascapular area which lasted for several days. Plain X-ray of the ribs and CT scan revealed an erosion of the left 11 th rib and a mass in the costodiaphragmatic recess. Both of these lesions were continuous with the lesion in left lung segment 10 . He was discharged without complaints after a two-month course of parenteral clindamycin, but continued treatment with oral clindamycin (300 $\mathrm{mg}$ tid). On follow-up radiological examinations, first pleural effusion and the lung consolidations disappeared, then the erosion of the rib disappeared, and finally, after six months on clindamycin, the mass in the costodiaphragmatic recess disappeared. He was last seen two years after completion of therapy, with no recurrence of disease observed.

\section{Discussion}

Disturbance of consciousness and poor oral hygiene are predisposing factors for anaerobic pleuropulmonary infection by aspiration. ${ }^{1}$ In our patient, both factors were present. In addition, continuity was noted between the polymicrobial anaerobic abscess in the chest wall and the lung parenchymal lesion, which disappeared during clindamycin therapy. Concerning the aetiology of the abscess, a prior skin lesion or a bone lesion which might have invaded the chest wall was excluded based on the anamnesis and the results of bone examination on admission. A septic chest wall abscess from a distant focus was hardly considered, because as many as five species of anaerobes were cultured. Pleural effusion was sterile. These considerations suggested that the abscess in the chest wall was caused by bacteria from the lung lesion, which was probably contracted by aspiration. Poorly controlled
Summary points

- disturbed consciousness and poor oral hygiene leading to aspiration are predisposing factors for anaerobic pulmonary infections

- in actinomycosis, pneumonia sometimes involves the thoracic wall without pleural empyema

- when encountering an anaerobic infection with multiple foci, it may be advisable to consider surgical intervention for the individual foci

diabetes can also predispose to infectious processes. $^{1}$ The rib erosion and the peridiaphragmatic mass appeared to have occurred in the same way, as they were near the lung lesion and disappeared during antibiotic therapy.

In the present case, two pathognomonic features of thoracic actinomycosis were present. Firstly, pulmonary disease had extended to the chest wall including a rib. Secondly, sulphur granules, which are conglomerations of filamentous rods, were found in the abscess. ${ }^{23}$ In this disease, there are two mechanisms by which the chest wall becomes involved. One is invasion from empyema, and the other is direct invasion from a subpleural parenchymal lesion, as in the present case. ${ }^{2-4}$ In the latter case, sterile pleural effusions sometimes occur, as in the patient described here. ${ }^{4}$ Thus, if the diagnosis cannot be made based on examination of the pleural effusion in patients presenting with a chest wall lesion and a pleural effusion, it is preferable to examine the lesion directly. ${ }^{25}$ In thoracic actinomycosis, chest wall lesions are said to consist mainly of granulomatous tissue with extensive fibrosis and small abscesses. ${ }^{2}{ }^{3}$ In this case, however, the lesion developed into a large abscess. Undoubtedly, the presence of other anaerobes influenced the patient's clinical course. ${ }^{2}$ In thoracic actinomycosis, other pathogens are often encountered, which are called 'associates'. ${ }^{2}{ }^{3}$

Drainage or surgical intervention is often required for the diagnosis and treatment of anaerobic infections including actinomycosis, because they tend to form abscesses. ${ }^{1}$ In the present case, the patient's condition improved rapidly after drainage of the abscess. However, we did not attempt to intervene with the peridiaphragmatic and bone lesions, because these interventions are not easy to perform; moreover, it was reasonable to assume that the lesions had been caused by the same organisms found in the chest wall abscess, and rapid clinical response had already been achieved with antibiotic therapy. During careful follow-up examination, the pain, probably due to the lesions, soon disappeared and both lesions completely disappeared during antibiotic therapy. When anaerobic infection with multiple foci is encountered, it may be advisable to consider surgical intervention of individual foci. It is preferable to prolong medical therapy beyond the resolution of measurable disease to prevent relapse, one of the clinical hallmarks of this infection. ${ }^{3}$ 


\section{Final diagnosis}

Thoracic actinomycosis.
Keywords: actinomycosis; thoracic wall; anaerobes; abscess
1 Finegold SM. Anaerobic bacteria: General concepts. In: Mandell GL, Bennett JE, Dolin R, eds. Principles and practice of infectious diseases. New York: Churchill Livingstone, 1995; pp 2156-73.

2 Heffner JE, Harley RA. Thoracic actinomycosis. Semin Respir Med 1992;13:234-41.

3 Russso TA. Agents of actinomycosis. In: Mandell GL, Bennett JE, Dolin R, eds. Principles and practice of infectious diseases. New York: Churchill Livingstone, 1995; pp 2280-8.
4 Kwong JS, Muller NL, Godwin JD, Aberle D, Grymaloski MR. Thoracic actinomycosis: CT findings in eight patients. Radiology 1992;183:189-92.

5 Pinto MM, Longstreth GB, Khoury GM. Fine needle aspiration of Actinomyces infection of the breast. A novel presentation of thoracopleural actinomycosis. Acta Cytol 1991; 35:409-11.

\title{
Haematemesis and chest pain in a middle-aged woman
}

\author{
P A Kitchen, M R Jacyna
}

A 57-year-old woman presented with acute-onset sharp retrosternal chest pain which occurred whilst walking. The pain radiated to her back and was associated with coffee-ground vomiting. She had eaten a meal two hours before with no discomfort. There was no previous history of ischaemic heart disease, hypertension, peptic ulcer disease or gastro-oesophageal reflux disease and she was not taking aspirin or any other nonsteroidal anti-inflammatory drug.

On examination she was well and had no surgical emphysema. The pulse was $90 \mathrm{beats} / \mathrm{min}$ and the blood pressure $150 / 85 \mathrm{mmHg}$ being equal in both arms. The respiratory and abdominal system were unremarkable. Routine biochemical and haematological parameters were normal apart from a haemoglobin of $11.8 \mathrm{~g} / \mathrm{dl}$ and a mean corpuscular volume of $88 \mathrm{fl}$. Her C-reactive protein (CRP) was $28 \mathrm{mg} / \mathrm{l}$. The chest X-ray and electrocardiogram were normal. She was then kept nil by mouth and intravenous fluids were started. She then had an urgent oesophagogastroduodenoscopy which showed a submucosal haematoma extending from 18-40 cm (figure 1). On retroversion of the endoscope there was an ulcerative area at the cardia, histology of which showed fibrinous debris but no evidence of malignancy. Computed tomography (CT) scan of the thorax showed a dilated proximal oesophagus with oesophageal wall thickening which narrowed to the level of the left pulmonary artery but no evidence of pathological lymph nodes or masses.

\section{Department of Gastroenterology, Northwick Park and St Marks Hospital NHS Trust, Watford Road, Harrow, Middlesex HA1 3UJ, UK P A Kitchen M R Jacyna}

Accepted 20 August 1997
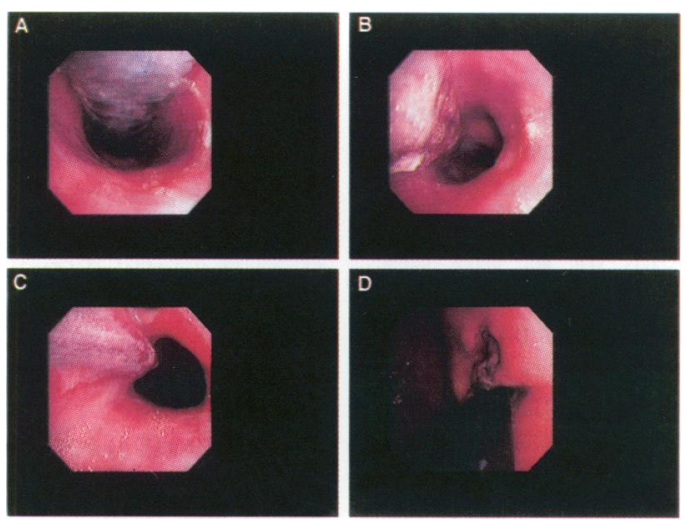

Figure 1 (A) Proximal portion of haematoma at 20 $\mathrm{cm}$; (B) view at $35 \mathrm{~cm}$; (C) distal portion at $40 \mathrm{~cm}$; (D) ulcerative area seen on retroverting the endoscope

\section{Questions}

1 What is the diagnosis?

2 What is the treatment? 